\title{
Precision Manufacture of Phase-Change Perfluorocarbon Droplets using Microfluidics
}

\author{
Thomas D. Martz ${ }^{1}$, Paul S. Sheeran², David Bardin ${ }^{3}$, Abraham P. Lee ${ }^{3}$, and Paul A. \\ Dayton ${ }^{2, *}$ \\ ${ }^{1}$ Curriculum of Applied Sciences and Engineering - Materials Science, The University of North \\ Carolina, Chapel Hill, NC, 27599 \\ 2Joint Department of Biomedical Engineering, The University of North Carolina and North \\ Carolina State University, Chapel Hill, NC, 27599
}

${ }^{3}$ Department of Biomedical Engineering, The University of California at Irvine, Irvine, CA, 92697

\begin{abstract}
Liquid perfluorocarbon droplets have been of interest in the medical acoustics community for use as acoustically activated particles for tissue occlusion, imaging, and therapeutics. To date, methods to produce liquid perfluorocarbon droplets typically result in a polydisperse size distribution. Since the threshold of acoustic activation is a function of diameter, there would be benefit from a monodisperse population to preserve uniformity in acoustic activation parameters. Through the use of a microfluidic device with flow focusing technology, the production of droplets of perfluoropentane with a uniform size distribution is demonstrated. Stability studies indicate that these droplets are stable in storage for at least two weeks. Acoustic studies illustrate the thresholds of vaporization as a function of droplet diameter, and a logarithmic relationship is observed between acoustic pressure and vaporization threshold within the size ranges studied. Droplets of uniform size have very little variability in acoustic vaporization threshold. Results indicate that microfluidic technology can enable greater manufacturing control of phase change perfluorocarbons for acoustic droplet vaporization applications.
\end{abstract}

\section{Keywords}

acoustic droplet vaporization; ultrasound; monodisperse; perfluoropentane; microfluidic

\section{Introduction}

\section{Acoustic Droplet Vaporization Agents}

In acoustic droplet vaporization (ADV), acoustic pressure delivered to a liquid droplet by an ultrasound (US) transducer provides the additional energy necessary to induce a liquid-togas phase transition. This phase transition allows for unique applications that are not possible with standard microbubble contrast agents, since ADV agents exhibit a very large

\footnotetext{
(C) 2011 World Federation for Ultrasound in Medicine and Biology. Published by Elsevier Inc. All rights reserved. *Corresponding Author: padayton@bme.unc.edu, Address: 304 Taylor Hall, CB 7575, Chapel Hill, NC 27599, Phone: (919) 843-9521, Fax: (919) 843-9520.

Publisher's Disclaimer: This is a PDF file of an unedited manuscript that has been accepted for publication. As a service to our customers we are providing this early version of the manuscript. The manuscript will undergo copyediting, typesetting, and review of the resulting proof before it is published in its final citable form. Please note that during the production process errors may be discovered which could affect the content, and all legal disclaimers that apply to the journal pertain.
} 
volume change after activation. Additionally, ADV agents are typically poorly echogenic in their liquid form, but highly echogenic as gas bubbles.

The basic construction of an ADV agent consists of a volatile liquid core that can be converted to a gas with additional energy. Typically, ADV droplets consist of perfluorocarbons (PFC) such as perfluoropentane (PFP)(Zhang et al. 2010), perfluorohexane (PFH)(Giesecke and Hynynen 2003, Fabiilli et al. 2010), or perfluorooctane (Fabiilli et al. 2009). The most commonly employed perfluorocarbons, PFP and PFH, have boiling points near physiological temperatures $\left(29^{\circ} \mathrm{C}\right.$ and $56{ }^{\circ} \mathrm{C}$, respectively). Recently, stabilization and ADV capability of droplets of liquid perfluorobutane (boiling point of $-1.7^{\circ} \mathrm{C}$ ) has been demonstrated (Sheeran et al. 2011).

Producing droplets in the micron and sub-micron range increases the Laplace pressure exerted on the liquid core, enabling small droplets to be maintained in liquid form even above their vaporization temperature. The addition of a stabilizing shell reduces droplet coalescence and increases stability of the emulsions. (Kripfgans et al. 2005, Rapoport et al. 2009a, Sheeran et al. 2011) Commonly utilized methods of producing PFC droplets include sonication or high-speed mechanical agitation of the host solution, which typically results in a polydisperse population (Fabiilli et al. 2010, Rapoport et al. 2010).

\section{Acoustic Droplet Vaporization Applications}

Liquid perfluorocarbon droplets have been shown to provide ultrasound image contrast (Kripfgans et al. 2002, Marsh et al. 2007), although the backscatter is notably less than similarly sized microbubble agents. One of the first commercial applications of perfluorocarbon droplets for ultrasound contrast enhancement was Echogen (Sonus Pharmaceuticals), a perfluoropentane emulsion (Quay 1996). Clinical trials for Echogen were abandoned in 2000 prior to FDA approval.

Once vaporized, ADV agents are readily detectable by an imaging system even at low concentrations. In very small amounts, droplets when converted to bubbles through ADV could act as point beacons for phase aberration corrections (Apfel 1998, Haworth et al. 2008). The unique advantages of ADV droplets in the hundred nanometer size range is that they have the potential to extravasate from the vasculature and into the tissue, enabling ADV based contrast imaging or therapy to extend beyond the vascular space, where traditional microbubbles cannot access (Rapoport et al. 2007, Rapoport et al. 2009b, Sheeran et al. 2011.).

One of the most well studied applications of ADV is embolotherapy. This technique enables the intentional creation of an occlusion in a specific tissue to limit blood flow. This has been proposed to treat renal cell carcinoma, to reduce blood loss for hypervascular tumors, and to treat hemhorrhage (Kripfgans et al. 2002, Zhang et al. 2010). Kripfgans et al. (2005) demonstrated that a filtered emulsion of droplets which is intra-arterially injected can reduce kidney perfusion by $70 \%$. This level of organ perfusion reduction has the ability to cause ischemic necrosis for therapeutic applications, or can enhance the utility of radiofrequency tissue ablation by minimizing thermally dissipative effects due to reduced flow (Kripfgans et al. 2005). A second application of ADV agents is site-specific therapeutics, where the goal is to deliver a potent agent only to the disease site while minimizing systemic effects. The ADV-mediated delivery of a chemotherapeutic drug directly to the tumor has been shown to mediate regression of ovarian and breast cancer (Rapoport et al. 2009a). ADV agents that have the ability to carry a therapeutic payload to a desired vessel could be combined with vessel occlusion upon activation to allow for an increase in residence time of the therapeutic agent (Fabiilli et al. 2010). 
With spatial and temporal control over ADV agent activation, ADV agents can be used as a nucleation site for high-intensity focused ultrasound (HIFU) tumor ablation. During HIFU, the microdroplets can be activated to increase the therapeutic effect or decrease the treatment time (Zhang and Porter 2010, Zhang et al. 2011).

\section{Considerations of ADV agent size distribution}

The size of ADV agents is an important factor in determining the vaporization threshold and the biodistribution. The Antoine equation predicts a negative logarithmic relation between droplet size and activation energy, where larger droplets require less energy to vaporize than smaller droplets (Rapoport et al. 2009b). Most who have characterized ADV-based agents have evaluated a polydisperse droplet population. While general trends in thresholds have been observed, polydispersity in droplet sizes has led to a variability in the vaporization threshold measured for studies that examine a bulk population of ADV droplets. Additionally, the larger droplets vaporize at lower energies and the resulting bubbles can influence the threshold energy for the smaller droplets (Lo et al. 2007), confounding in-vitro characterization of vaporization thresholds. Since microbubbles combined with high acoustic pressures can lead to unwanted bioeffects (Miller et al. 2008), it may be desirable to perform $\mathrm{ADV}$ at the minimum acoustic pressure required. A polydisperse sample may require a higher pressure to efficiently vaporize the entire population due to smaller droplets requiring more energy.

For micron-sized ADV agents, one of the primary applications is vessel occlusion, where it may be important to have a well characterized distribution of droplet sizes to target a specific vascular diameter while not affecting other vessels (Kripfgans et al. 2005). For the same reasons, a droplet designed for imaging applications that is too large could lodge in the lungs or other organs after IV injection, leading to undesired vessel occlusion. All of these limitations are motivating reasons for precise size control of ADV agents.

Previous studies to improve the size distribution characteristics of perfluorocarbon droplets have involved microfluidic sorting (Huh et al. 2007) and filtering (Kripfgans et al. 2005). The microfluidics platform is well known for the ability to create particles (Xu et al, 2005), droplets (Tan et al. 2004, Xu et al. 2006, Teh et al. 2008), microbubbles (Hettiarachchi et al. 2007), and multi-layer/multi component compositions (Hettiarachchi et al. 2009, Wang et al. 2011) of uniform size. In this manuscript, we assess the feasibility for producing a monodispersed ADV agent through a microfluidic technique, in order to achieve uniform activation thresholds.

\section{Materials and Methods Reagent Preparation}

Liquid Perfluoropentane was purchased from FluoroMed, L.P. (Round Rock, Texas). Lipid solutions were created using a 9:1 molar ratio of 1,2-distearoyl-sn-glycero-3-phosphocholine (DSPC) (Avanti Polar Lipids, Alabaster, AL) and 1,2-distearoyl-sn-glycero-3phosphoethanolamine-N[methoxy-(polyethylene glycol)-2000] (DSPE-PEG2000) (Avanti Polar Lipids, Alabaster, AL) in a $110 \mathrm{~mL}$ solution of deionized water (DI), glycerol (Fisher Scientific, Pittsburgh, PA), propylene glycol (Fisher Scientific, Pittsburgh, PA), and pluronic F-68 (Sigma-Aldrich Corporation, St. Louis, MO).

$50 \mathrm{mg}$ of DPSC and $19.6 \mathrm{mg}$ DSPE-PEG2000 were dissolved in chloroform. The chloroform was then evaporated with $\mathrm{N}_{2}$, after which $50 \mathrm{~mL}$ of DI was added and the resulting solution was sonicated for 20 minutes. After sonication, $47 \mathrm{~mL}$ DI, $2 \mathrm{~mL}$ glycerol, 
$1 \mathrm{~mL}$ propylene glycol, and $10 \mathrm{~mL}$ pluronic F-68 were added. The solution was vortex mixed for 1 minute and then sonicated for 10 minutes.

\section{Microfluidic Device Fabrication}

The process for developing the microfluidic wafer has been previously described by Hettiarachchi el al. (2009). Briefly, the channels were created using Illustrator (Adobe, San Jose, CA) and printed (20,000 dpi) by CAD/Art Services (Brandon, OR). In a clean room, the wafer was first oxidized in oxygen plasma then spin-coated with a UV curable epoxy (SU-8-25, MicroChem, Newton, MA). On exposing the wafer to UV light, the channel patterns develop to use as a negative for device production.

With the wafer in a petri dish, Polydimethylsiloxane (PDMS) (Dow Corning, Midland, MI) and a curing agent were poured over the wafer at a 10:1 ratio, respectively. This was then placed under vacuum for 24 hours and then cured at $60^{\circ} \mathrm{C}$ overnight. After the PDMS was cured, the individual microfluidic devices were removed from the wafer. The individual microfluidic device and a clean microscope slide (2947-75×25, Corning, Corning, NY) were placed in a plasma cleaner (Harrick Plasma, Ithaca, NY) and exposed to a plasma field under a controlled pressure of 500 millitorr for 3 minutes. On completion of the plasma cleaning process, the PDMS and glass slide were immediately joined, forming complete microchannels. One minute after the bond was established, DI water was injected into the channels to create a hydrophilic environment.

\section{Microfluidic Experimental Apparatus}

The microfluidic experimental apparatus was set up similar to that as described previously by Kaya et al. (2010). Briefly, the microfluidic device was secured to a moving stand on an inverted microscope (Olympus $1 \times 71$ microscope, Center Valley, PA), and viewed with a $50 \times \mathrm{NA}=0.5$ objective. A high speed camera (Fastcam APX-RS, Photron, Inc., San Diego, CA) was used to capture images and videos of the device output. Reagents were injected from syringes into device channels through Tygon S-54-HL tubing (Saint-Gobain Performance Plastics, Akron, $\mathrm{OH}$ ). Calibrated syringe pumps (Harvard Apparatus, Holliston, MA) were used to control rate of material flow in individual microchannels.

\section{Sample Production}

To produce uniform samples, the PFP flow rate was fixed at a constant value and the lipid flow rate was increased until the smallest stable droplets were formed. The system was allowed to stabilize for 5 minutes before collection, since pressure changes in the system required a few minutes to equalize. This process was repeated across a range of different flow rates, as described in Table 1. In order to determine the production rate for the microfluidic device, high-speed video analysis of the droplet production was performed. Time stamps enabled the determination of droplet production rate.

\section{Acoustic Experimental Apparatus}

The experimental setup to determine droplet vaporization thresholds was similar to that previously described by (Sheeran et al. 2011). Briefly, a heated water bath maintained at $37^{\circ} \mathrm{C}$ was connected to an inverted microscope. Droplets were injected through a nearly acoustically and optically transparent microcellulose capillary tube with an inner diameter of $200 \mu \mathrm{m}$ (Spectrum Labs, Inc., Greensboro, NC). A 5 MHz piston transducer (IL0506HP, Valpey Fisher Corp., Hopkinton, MA) was positioned in the water bath confocally with a water immersion microscope objective $(100 \times, \mathrm{NA}=1.0)$. The output of the ultrasound transducer was calibrated by inserting a needle-hydrophone (HNA-0400, Onda Corp., Sunnyvale, CA) into the experimental system at the exact location where the samples are 
positioned during activation studies (the focus of the microscope objective). Droplets were exposed to single 10-cycle sinusoidal pulses, while the ultrasound pressure was increased incrementally until droplet vaporization was observed. The procedure was repeated for at least 20 droplets from each size distribution to verify droplet size and threshold.

\section{Stability Analysis}

Droplets were stored at $4^{\circ} \mathrm{C}$ in $3 \mathrm{~mL}$ vials until sampling. For analysis, a 25 microliter aliquot of droplets was placed on a glass slide, and images were captured with the Photron Fastcam camera. Size measurements were performed in Image J (NIH, Bethesda, MD) by comparison to a calibrated reticle (Edmund Industrial Optics, Barrington, NJ). A minimum of 50 droplets were measured for each population.

\section{Results}

The dimensions of the microfluidic device, material composition, and flow rates all have the ability to alter the composition of the resulting droplet. Due to the microfabrication process, there are small variations from device to device (we measured a 13\% deviation in orifice widths across 10 devices). With this in mind, only one device was used for the duration of production to ensure that the dimensions of the device were unchanged. The material compositions also went unchanged to ensure that the droplets would experience a standardized reaction to the US pressure.

\section{Precision Size Control}

The flow rates were varied over five sets of conditions for the duration of the experiment to alter the droplet diameter. Results illustrated that by varying flow rates, the PFP population diameters could be carefully selected. In general, as the lipid flow rate was increased, droplet size decreased (Figure 1). As the PFP flow rate was increased, droplet size increased. Table 1 lists the flow rates, mean droplet size, standard deviation, and polydispersity index (Xu et al. 2006).

For the populations studied, the standard deviation was largest for the smallest droplets ( $\mathrm{SD}=0.4$ for the 7.4 micron population) and smallest for the largest droplets $(0.3$ for the 20.4 micron population). All populations had a polydispersity index of 5.1 or less.

\section{Production Rate}

The $20-\mu \mathrm{L} / \mathrm{min}$ lipid and the $12.5 \mu \mathrm{L} / \mathrm{min}$ PFP flow rates, which resulted in 20.4 micron droplets, had a production rate of $1.85 \times 10^{4}$ droplets produced per second (slowest production sample); whereas the $60 \mu \mathrm{L} / \mathrm{min}$ lipid and $13.9 \mu \mathrm{L} / \mathrm{min}$ PFP flows, which produced 7.4 micron droplets, had a production rate of $1.65 \times 10^{5}$ droplets per second (fastest production sample). Table 2 lists the production rates for the 5 samples.

\section{Acoustic Responses}

The five samples were diluted and injected into a cellulose microcapillary tube where droplets from each population were individually subjected to US. The US data showed that there was a distinct vaporization threshold pressure for each diameter (Figure 2). The data trend suggests a logarithmic relationship $\left(P=-1.80 \ln (D)+7.72, R^{2}=0.97\right)$, as predicted by the Antoine equation.

\section{Long-tem stability}

Populations maintained a fairly low polydispersity over a two week time period (Table 1), and overall, the mean diameters of the populations drifted less than $4.6 \%$ (Figure 3 ). The 
16.2 and $10.3 \mu \mathrm{m}$ mean diameter populations each maintained the same polydispersity, and the $20.4,12.6$, and $7.4 \mu \mathrm{m}$ mean diameter populations changed by a factor of $1.8,1.3$, and 1.9 , respectively.

\section{Discussion}

The method described here demonstrates the potential of production of uniform-sized droplets for acoustic droplet vaporization utilizing microfluidics. Data illustrates that this technique enabled production of a population of droplets where each droplet responded similarly to the same acoustic parameters due to uniform size. One advantage of utilizing such uniform droplets would include the ability to use the lowest acoustic pressure possible for vaporization (since all droplets would respond similarly), thereby achieving the highest vaporization efficiency for the lowest acoustic pressure. Keeping the acoustic pressure as low as required would conform to the "As Low As Reasonably Achievable (ALARA) Principle" for clinical ultrasonics. Other potential advantages of uniform sized ADV agent production, although not demonstrated here, would be the ability to tailor droplet sizes for specific vessel occlusion, or to enable precision drug-loading for therapeutic-carrying ADV agents.

Microfluidic techniques have previously demonstrated unique utility in the controlled fabrication of droplets and microbubbles. The biggest limitation to this method to date is that the production rate is substantially slower than other droplet and microbubble production methods. Even for the fastest production method here, it would require nearly 3 hours to achieve $1 \times 10^{9}$ droplets (a similar order of magnitude to the concentration of microbubble contrast agents prepared by a mechanical agitation method). However, in the future microfluidic techniques may be scaled up to achieve higher throughput, although the need to maintain identical dimensions and flow rates to each orifice in order to preserve size uniformity presents a challenge. It should also be noted that although the smallest droplets described here were approximately 7 microns, which is likely too large for in-vivo application, this size was limited only by our particular system, and is not a fundamental limit of microfluidic droplet production. It is very reasonable to anticipate that by optimizing the channel dimensions, component flow rates, and the lipid/perfluorocarbon ratios, droplets much smaller than 7 microns can be prepared. Prior studies have illustrated microfluidic production of liquid droplets as small as 100 nanometers (Tan and Lee, 2005).

\section{Conclusion}

A microfluidic device can produce monodisperse PFP/lipid droplets using flow focusing technology. Monodisperse PFP/lipid droplets that were subjected to US pressure waves showed a uniform ADV response. The relationship formed by droplet diameter and ADV threshold agreed with the expected negative logarithmic response based on the size of the droplets. Droplet populations produced with microfluidics were stable within the two week time period studied.

\section{Acknowledgments}

The authors would like to thank Jason Streeter for the preparation of the lipid solution and Roger Shih for the microfluidic wafer fabrication. Also, the authors appreciate the assistance from Dennis Given and Roshni Kothadia for their work in droplet characterization and data organization. This project was supported by R01EB008733 and R21EB011704. 


\section{References}

Apfel, RE. Activatable infusable dispersions containing drops of a superheated liquid for methods of therapy and diagnosis. United States: Apfel Enterprises, Inc.; 1998.

Fabiilli ML, Lee JA, Kripfgans OD, Carson PL, Fowlkes JB. Delivery of water-soluble drugs using acoustically triggered perfluorocarbon double emulsions. Pharm Res. 2010; 27:2753-65. [PubMed: 20872050]

Fabiilli ML, Haworth KJ, Fakhri NH, Kripfgans OD, Carson PL, Fowlkes JB. The Role of Inertial Cavitation in Acoustic Droplet Vaporization. Ieee Transactions on Ultrasonics Ferroelectrics and Frequency Control. 2009; 56:1006-17.

Giesecke T, Hynynen K. Ultrasound-mediated cavitation thresholds of liquid perfluorocarbon droplets in vitro. Ultrasound Med Biol. 2003; 29:1359-65. [PubMed: 14553814]

Haworth KJ, Fowlkes JB, Carson PL, Kripfgans OD. Towards aberration correction of transcranial ultrasound using acoustic droplet vaporization. Ultrasound Med Biol. 2008; 34:435-45. [PubMed: 17935872]

Hettiarachchi K, Talu E, Longo ML, Dayton PA, Lee AP. On-chip generation of microbubbles as a practical technology for manufacturing contrast agents for ultrasonic imaging. Lab Chip. 2007; 7:463-8. [PubMed: 17389962]

Hettiarachchi K, Zhang S, Feingold S, Lee AP, Dayton PA. Controllable microfluidic synthesis of multiphase drug-carrying lipospheres for site-targeted therapy. Biotechnol Prog. 2009; 25:938-45. [PubMed: 19455647]

Huh D, Bahng JH, Ling Y, Wei HH, Kripfgans OD, Fowlkes JB, Grotberg JB, Takayama S. Gravitydriven microfluidic particle sorting device with hydrodynamic separation amplification. Anal Chem. 2007; 79:1369-76. [PubMed: 17297936]

Kaya M, Feingold S, Hettiarachchi K, Lee AP, Dayton PA. Acoustic responses of monodisperse lipidencapsulated microbubble contrast agents produced by flow focusing. Bubble Sci Eng Technol. 2010; 2:33-40. [PubMed: 21475641]

Kripfgans OD, Fowlkes JB, Woydt M, Eldevik OP, Carson PL. In vivo droplet vaporization for occlusion therapy and phase aberration correction. IEEE Trans Ultrason Ferroelectr Freq Control. 2002; 49:726-38. [PubMed: 12075966]

Kripfgans OD, Orifici CM, Carson PL, Ives KA, Eldevik OP, Fowlkes JB. Acoustic droplet vaporization for temporal and spatial control of tissue occlusion: A kidney study. Ieee Transactions on Ultrasonics Ferroelectrics and Frequency Control. 2005; 52:1101-10.

Lo AH, Kripfgans OD, Carson PL, Rothman ED, Fowlkes JB. Acoustic droplet vaporization threshold: effects of pulse duration and contrast agent. IEEE Trans Ultrason Ferroelectr Freq Control. 2007; 54:933-46. [PubMed: 17523558]

Marsh JN, Partlow KC, Abendschein DR, Scott MJ, Lanza GM, Wickline SA. Molecular imaging with targeted perfluorocarbon nanoparticles: quantification of the concentration dependence of contrast enhancement for binding to sparse cellular epitopes. Ultrasound Med Biol. 2007; 33:950-8. [PubMed: 17434667]

Miller DL, Averkiou MA, Brayman AA, Everbach EC, Holland CK, Wible JH Jr, Wu J. Bioeffects considerations for diagnostic ultrasound contrast agents. J Ultrasound Med. 2008; 27:611-32. quiz 33-6. [PubMed: 18359911]

Quay, SC. Phase shift colloids as ultrasound contrast agents. United States: Sonus Pharmaceuticals; 1996.

Rapoport N, Christensen DA, Kennedy AM, Nam KH. Cavitation Properties of Block Copolymer Stabilized Phase-Shift Nanoemulsions Used as Drug Carriers. Ultrasound in Medicine and Biology. 2010; 36:419-29. [PubMed: 20133040]

Rapoport NY, Kennedy AM, Shea JE, Scaife CL, Nam KH. Controlled and targeted tumor chemotherapy by ultrasound-activated nanoemulsions/microbubbles. J Control Release. 2009a; 138:268-76. [PubMed: 19477208]

Rapoport NY, Efros AL, Christensen DA, Kennedy AM, Nam KH. Microbubble Generation in PhaseShift Nanoemulsions used as Anticancer Drug Carriers. Bubble Sci Eng Technol. 2009b; 1:31-39. [PubMed: 20046899] 
Rapoport N, Gao Z, Kennedy A. Multifunctional nanoparticles for combining ultrasonic tumor imaging and targeted chemotherapy. J Natl Cancer Inst. 2007; 99:1095-106. [PubMed: 17623798]

Sheeran PS, Wong VP, Luois S, McFarland RJ, Ross WD, Feingold S, Matsunaga TO, Dayton PA. Decafluorobutane as a phase-change contrast agent for low-energy extravascular ultrasound imaging. Ultrasound Med Biol. 2011 E-pub:1-13.

Tan YC, Fisher JS, Lee AI, Cristini V, Lee AP. Design of microfluidic channel geometries for the control of droplet volume, chemical concentration, and sorting. Lab Chip. 2004; 4:292-8. [PubMed: 15269794]

Tan YC, Lee AP. Microfluidic separation of satellite droplets as the basis of a monodispersed micron and submicron emulsification system. Lab Chip. 2005; 5:1178-83. [PubMed: 16175277]

Teh SY, Lin R, Hung LH, Lee AP. Droplet microfluidics. Lab Chip. 2008; 8:198-220. [PubMed: 18231657]

Wang W, Xie R, Ju XJ, Luo T, Liu L, Weitz DA, Chu LY. Controllable microfluidic production of multicomponent multiple emulsions. Lab Chip. 2011; 11:1587-92. [PubMed: 21461409]

Xu S, Nie Z, Seo M, Lewis P, Kumacheva E, Stone HA, Garstecki P, Weibel DB, Gitlin I. Whitesides GM Generation of monodisperse particles by using microfluidics: control over size, shape, and composition. Angew Chem Int Ed Engl. 2005; 44:724-8. [PubMed: 15612064]

$\mathrm{Xu}$ JH, Li SW, Tan J, Wang YJ, Luo GS. Controllable preparation of monodisperse O/W and W/O emulsions in the same microfluidic device. Langmuir. 2006; 22:7943-6. [PubMed: 16952223]

Zhang M, Fabiilli ML, Haworth KJ, Fowlkes JB, Kripfgans OD, Roberts WW, Ives KA, Carson PL. Initial investigation of acoustic droplet vaporization for occlusion in canine kidney. Ultrasound Med Biol. 2010; 36:1691-703. [PubMed: 20800939]

Zhang P, Porter T. An in vitro study of a phase-shift nanoemulsion: a potential nucleation agent for bubble-enhanced HIFU tumor ablation. Ultrasound Med Biol. 2010; 36:1856-66. [PubMed: 20888685]

Zhang, M.; Fabiilli, ML.; Haworth, KJ.; Padilla, F.; Swanson, SD.; Kripfgans, OD.; Carson, PL.; Fowlkes, JB. Acad Radiol. 2011. Acoustic Droplet Vaporization for Enhancement of Thermal Ablation by High Intensity Focused Ultrasound. 


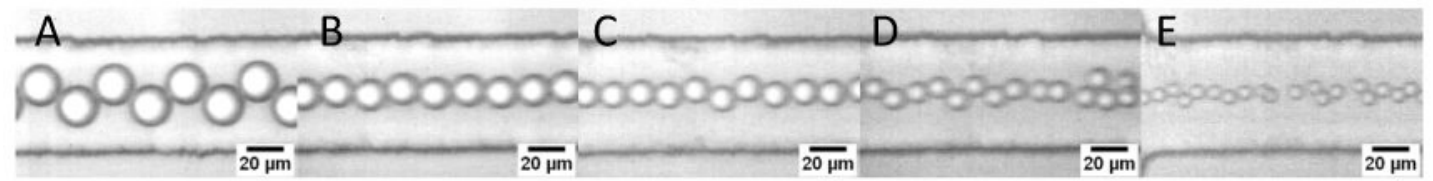

Figure 1.

High speed video frames of microfluidic droplet production at diameters of (A) $20.4 \pm 0.3$ $\mu \mathrm{m}$, (B) $16.2 \pm 0.5 \mu \mathrm{m}$, (C) $12.6 \pm 0.5 \mu \mathrm{m}$, (D) $10.3 \pm 0.4 \mu \mathrm{m}$, and (E) $7.4 \pm 0.4 \mu \mathrm{m}$, corresponding to lipid and PFP flow rates of lipid $40 \mu \mathrm{L} / \mathrm{min}$; PFP $12.5 \mu \mathrm{L} / \mathrm{min}$, lipid 31 $\mu \mathrm{L} / \mathrm{min}$; PFP $15 \mu \mathrm{L} / \mathrm{min}$, lipid $40 \mu \mathrm{L} / \mathrm{min}$; PFP $15 \mu \mathrm{L} / \mathrm{min}$, lipid $50 \mu \mathrm{L} / \mathrm{min}$; PFP $15 \mu \mathrm{L} /$ min, and lipid $60 \mu \mathrm{L} / \mathrm{min}$; PFP $13.9 \mu \mathrm{L} / \mathrm{min}$, respectively. 


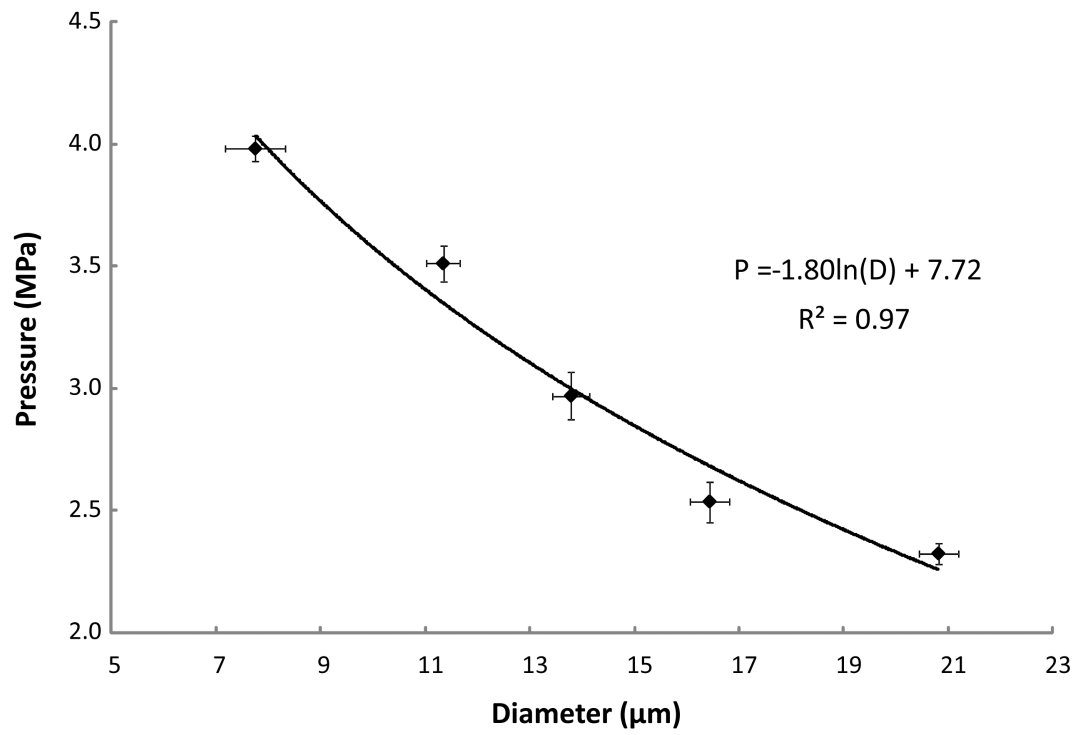

Figure 2.

Average pressure of vaporization vs. average droplet diameter. Error bars indicate standard deviations in pressure and diameter. The trendline is logarithmic. 


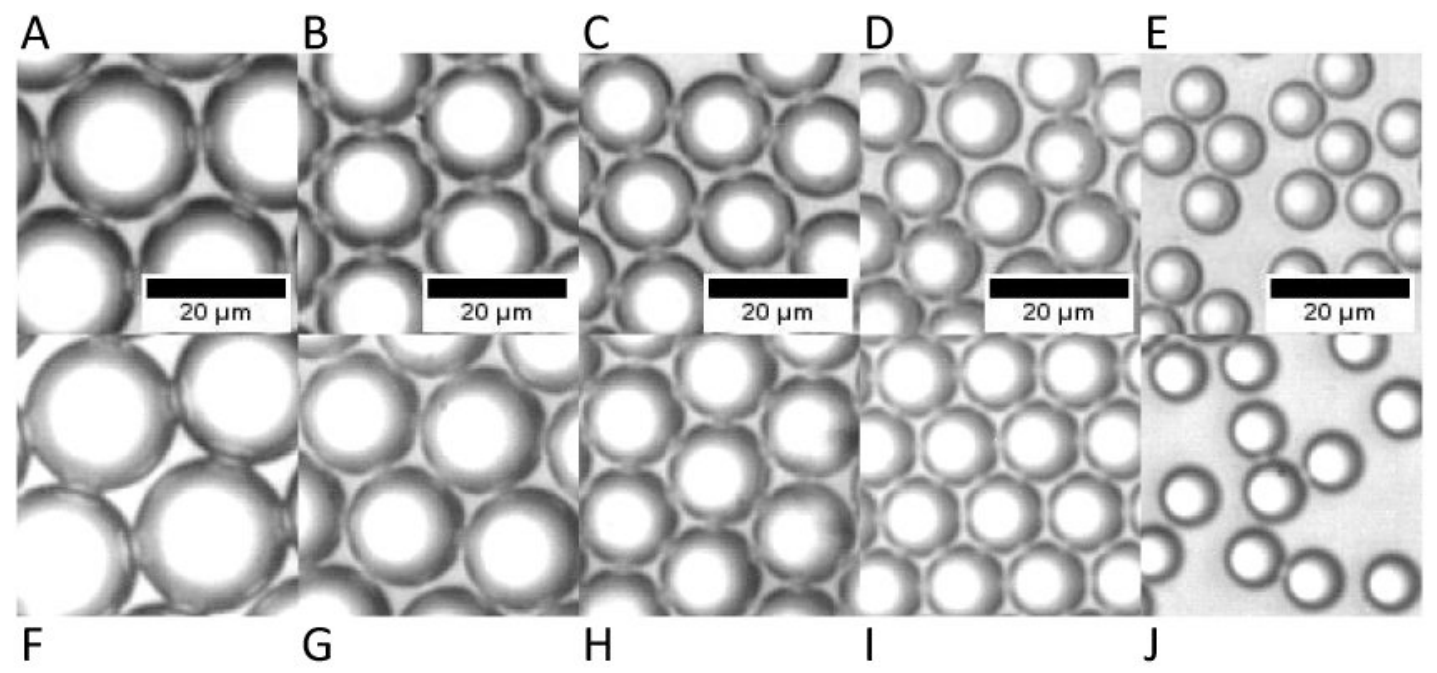

Figure 3.

Microscopy images of droplets 1 week apart illustrating size stability. (A-E) 1 week after production - populations of mean diameter of (A) $20.4 \pm 0.3 \mu \mathrm{m}$, (B) $16.2 \pm 0.5 \mu \mathrm{m}$, (C) $12.6 \pm 0.5 \mu \mathrm{m}$, (D) $10.3 \pm 0.4 \mu \mathrm{m}$, and (E) $7.4 \pm 0.4 \mu \mathrm{m}$. (F-J) 2 weeks after production populations corresponding to (A-E), respectively, with mean diameters of (F) $20.4 \pm 0.5 \mu \mathrm{m}$, (G) $15.5 \pm 0.5 \mu \mathrm{m}$, (H) $12.2 \pm 0.4 \mu \mathrm{m}$, (I) $10.8 \pm 0.4 \mu \mathrm{m}$, and (J) $7.5 \pm 0.7 \mu \mathrm{m}$. 


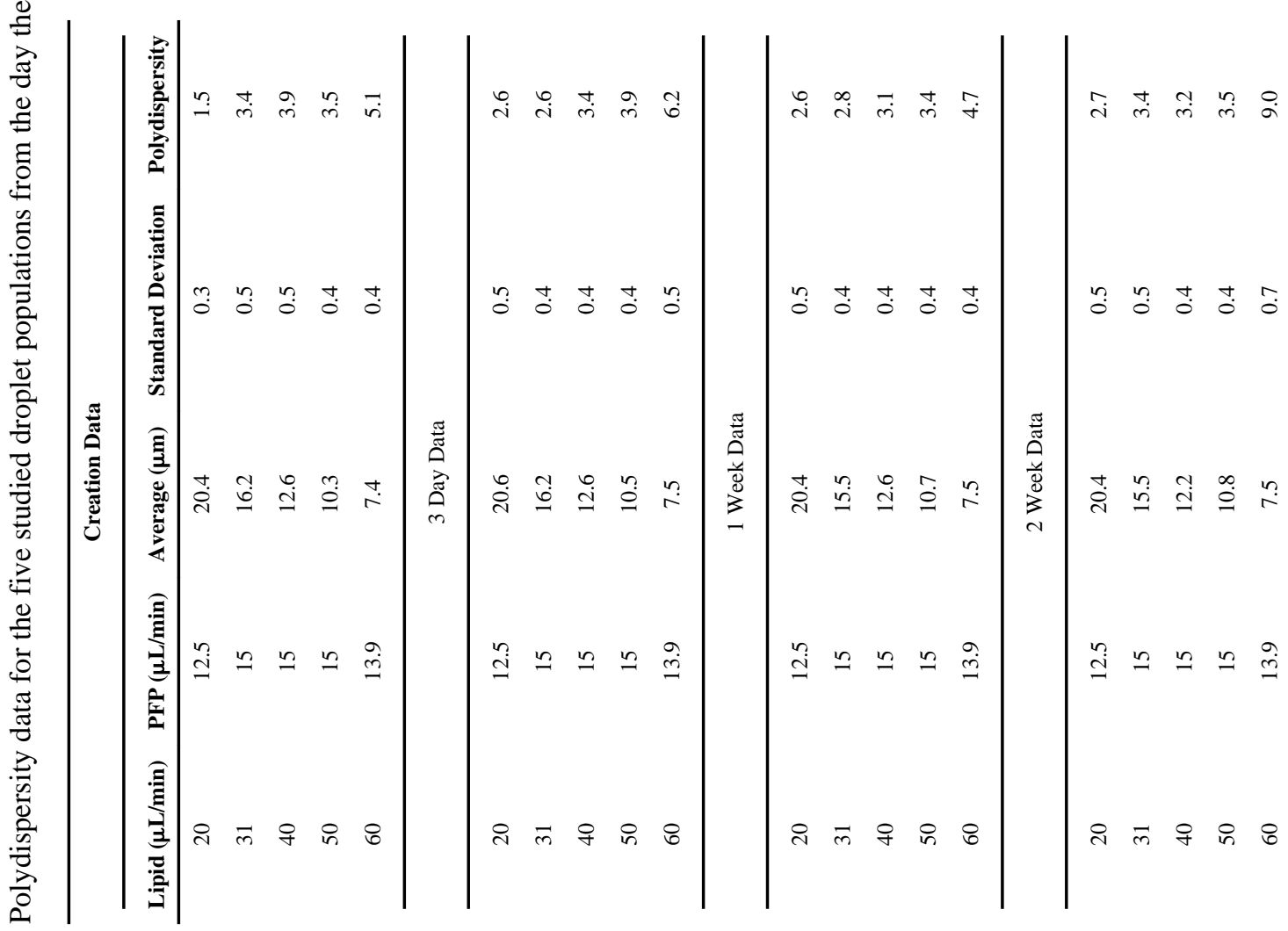




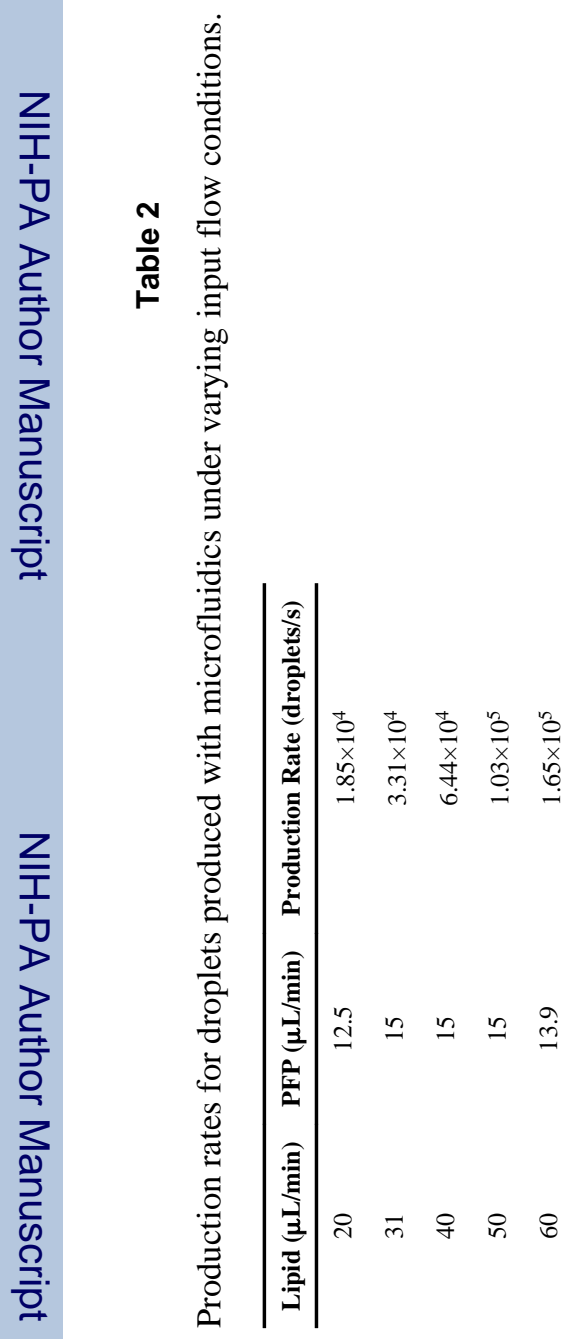

